10,04

\title{
Применение теории случайных матриц для описания бозонного пика в двумерных системах
}

\author{
(С) Д.А. Конюх ${ }^{1,2}$, Я.М. Бельтюков ${ }^{1}$ \\ ${ }^{1}$ Физико-технический институт им. А.Ф. Иофрфе РАН, \\ Санкт-Петербург, Россия \\ ${ }^{2}$ Санкт-Петербургский политехнический университет, \\ Санкт-Петербург, Россия \\ E-mail: conyuh.dmitrij@yandex.ru
}

Поступила в Редакцию 9 декабря 2019 г.

В окончательной редакции 9 декабря 2019 г.

Принята к публикации 10 декабря 2019 г.

Применена теория случайных матриц для описания колебательных свойств двумерных неупорядоченных систем с большим числом степеней свободы. Показано, что коррелированный ансамбль Вишарта позволяет учесть наиболее значимые механические свойства аморфных твердых тел. В данном ансамбле наблюдается избыточная плотность колебательных состояний по сравнению с законом Дебая, что выражается в пике в приведенной плотности состояний $g(\omega) / \omega$. Такой пик известен как бозонный пик, наблюдаемый во многих экспериментах и численных расчетах по изучению двумерных и трехмерных неупорядоченных систем. Показано, что двумерные системы имеют ряд отличий в асимптотическом поведении бозонного пика.

Ключевые слова: аморфные тела, бозонный пик, случайные матрицы.

DOI: 10.21883/FTT.2020.04.49127.645

\section{1. Введение}

Аморфные твердые тела обладают рядом отличительных свойств по сравнению с кристаллами. Одной из наиболее важных особенностей аморфных тел является присутствие так называемого бозонного пика. Данное явление заключается в наличии избыточной плотности колебательных состояний по сравнению с законом Дебая, согласно которому низкочастотная плотность состояний пропорциональна $\omega^{d-1}$, где $d-$ размерность пространства [1]. Пик, который наблюдается в приведенной плотности состояний $g(\omega) / \omega^{d-1}$ и получил название бозонного пика. Бозонный пик проявляется в ряде экспериментальных работ: в комбинационном рассеянии света [2], в неупругом рентгеновском рассеянии [3], в неупругом рассеянии нейтронов [4] и при измерении теплоемкости [5]. Несмотря на большое число таких работ, посвященных наблюдению бозонного пика, его природа остается не полностью изученной.

Открытым вопросом остается отличие бозонного пика в двумерных и трехмерных неупорядоченных системах. Двумерные системы широко исследуются численными методами [6-8] поскольку такие системы требуют меньше вычислительных ресурсов по сравнению с трехмерными системами. Кроме того, существует ряд экспериментальных работ, посвященных непосредственной регистрации колебаний частиц в двумерных гранулярных системах [9-10]. Бозонный пик также наблюдался в экспериментах по изучению спектра аморфных тел методом рассеяния атомов гелия на поверхности аморфного кремния [11].
Интересной особенностью бозонного пика является наличие связи между частотой бозонного пика $\omega_{b}$ (частотой, при которой достигается максимум приведенной плотности состояний $\left.g(\omega) / \omega^{d-1}\right)$ и упругими свойствами аморфной среды. Линейная связь между $\omega_{b}$ и модулем сдвига $G$ наблюдалась в экспериментах с различными модификациями боратов стекол $[5,12]$ и в работах по изучению распространения колебаний в гранулярных средах [13] вблизи так называемой ,jamming transition point“". Однако данная зависимость до сих пор не имеет теоретического обоснования, ее существование в двумерных системах носит открытый характер. Поэтому понимание отличия колебательных свойств двумерных и трехмерных систем играет важную роль в интерпретации полученных результатов.

Малые колебания атомов с единичными массами $\left(m_{i}=1\right)$ могут быть описаны с помощью динамической матрицы $\hat{M}$ как

$$
\ddot{u}_{i}=-\sum_{j} M_{i j} u_{j}
$$

где $u_{i}$ - смещение, описывающее колебание $i$-ой степени свободы. Динамическая матрица связана с полной потенциальной энергией межатомного взаимодействия $U\left(u_{1}, u_{2}, \ldots\right)$ следующим соотношением:

$$
M_{i j}=\frac{\partial^{2} U}{\partial u_{i} \partial u_{j}} .
$$

В аморфных телах динамическую матрицу $\hat{M}$ можно считать до некоторой степени случайной матрицей. 
Поэтому целью нашей работы является применение теории случайных матриц для анализа колебательных свойств аморфных твердых тел. Теория случайных матриц имеет важные приложения во многих различных областях науки и техники при анализе сложных систем, состоящих из большого числа степеней свободы [14-24]. Однако исследуемая колебательная система имеет ряд отличительных особенностей. Во-первых, система должна находиться вблизи устойчивого положения равновесия, что означает положительную определенность динамической матрицы $\hat{M}$ [25]. Во-вторых, колебания системы не зависят от сдвига всей системы как целого, в результате чего получается правило сумм $\sum_{i} M_{i j}=\sum_{j} M_{i j}=0$.

Заметим, что любую положительно определенную матрицу $\hat{M}$ можно всегда представить в виде $\hat{M}=\hat{A} \hat{A}^{T}$, где $\hat{A}-$ некоторая матрица размером $N \times K$. И наоборот, матрица вида $\hat{A} \hat{A}^{T}$ всегда положительно определена и тем самым задает устойчивую механическую систему. Действительно, в этом случае энергия системы записывается в виде суммы квадратичных форм

$$
U=\frac{1}{2} \sum_{j}\left(\sum_{i} A_{i j} u_{i}\right)^{2},
$$

где индекс $i$ нумерует степени свободы, а индекс $j-$ квадратичные формы. Каждую из таких квадратичных форм, которой соответствует $j$-й столбец матрицы $\hat{A}$, можно считать „связью“ некоторого числа степеней свободы между собой [26].

Таким образом, задание матрицы $\hat{A}$ не требует учета механической устойчивости системы, поскольку динамическая матрица $\hat{M}=\hat{A} \hat{A}^{T}$ автоматически удовлетворяет этому свойству. Существующие подходы с применением случайных матриц для описания колебаний не используют форму $\hat{A} \hat{A}^{T}$, которая гарантирует устойчивость системы, что не позволяет варьировать беспорядок в достаточно широких пределах $[27,28]$. Ансамбль случайных матриц вида $\hat{A} \hat{A}^{T}$ получил название ансамбля Вишарта. В первом приближении матрицу $\hat{A}$ можно считать случайной матрицей с независимыми матричными элементами. В таком случае плотность колебательных состояний подчиняется закону Марченко-Пастура, что позволяет качественно описать доминирующий диапазон частот $[29,30]$. Однако исследование низкочастотной области, в том числе области бозонного пика, требует удовлетворения правила сумм для динамической матрицы. Нарушение правила сумм приводит к отсутствию низкочастотных колебаний в виде плоских волн и нарушению закона Дебая [31]. Заметим, что каждая связь (квадратичная форма) в уравнении (3) должна удовлетворять правилу сумм, в результате чего $\sum_{i} A_{i j}=0$. Таким образом, необходимо учитывать корреляции между элементами матрицы $\hat{A}$.

Для простоты будем считать, что все связи статистически одинаковы и независимы. Другими словами, отдельные столбцы матрицы $\hat{A}$ не коррелируют друг с другом. В этом случае парные корреляции между матричными элементами $A_{i j}$ можно записать в виде

$$
\left\langle A_{i j} A_{k l}\right\rangle=\frac{1}{N} C_{i k} \delta_{j l}
$$

где $\hat{C}$ - некоторая корреляционная матрица. Можно заметить, что $\hat{C}=\frac{N}{K}\langle\hat{M}\rangle$, где $\langle\hat{M}\rangle-$ усредненная по ансамблю динамическая матрица. Для простоты рассмотрим аморфную систему в виде простой квадратной решетки со случайными связями. В этом случае средняя динамическая матрица $\langle\hat{M}\rangle$ является кристаллической матрицей. Естественно предположить, что кристаллическая матрица имеет простые связи между ближайшими соседями с определенной жесткостью. Мы будем рассматривать так называемую скалярную модель, в которой каждая частица обладает только одной степенью свободы. В этом случае корреляционная матрица $\hat{C}$ имеет следующую структуру. Недиагональные элементы $C_{i j}=-\Omega^{2}$, если атомы с индексами $i$ и $j$ являются ближайшими соседями в решетке. В противном случае $C_{i j}=0$. Диагональные элементы $C_{i i}=4 \Omega^{2}$. Константа $\Omega$ определяет характерную частоту в системе.

Введем параметр $\varkappa=K / N-1$, который показывает соотношение числа степеней свободы (число строк матрицы $\hat{A}$ ) и числа связей в системе (число столбцов матрицы $\hat{A}$ ). Если число связей $K$ существенно превосходит число степеней свободы $N$, то $\varkappa \gg 1$ и динамическая матрица $\hat{M}$ слабо отличается от усредненной матрицы $\langle\hat{M}\rangle$. При уменьшении параметра $\varkappa$ растут флуктуации элементов динамической матрицы. Таким образом, параметр $\varkappa$ характеризует отношение порядка и беспорядка в системе. В первую очередь нас будут интересовать существенно разупорядоченные системы для которых $\varkappa \ll 1$.

\section{2. Плотность колебательных состояний}

Собственные числа динамической матрицы $\hat{M}$ являются квадратами собственных частот $\omega_{i}^{2}$. Для нахождения распределения собственных частот (плотности колебательных состояний) $g(\omega)$ будем считать, что динамическая матрица $\hat{M}=\hat{A} \hat{A}^{T}$ подчиняется коррелированному ансамблю Вишарта. В таком ансамбле элементы матрицы $\hat{A}$ подчиняются многомерному гауссову распределению с корреляциями, описываемыми уравнением (4). При этом средние значения элементов матрицы $\hat{A}$ равны нулю, поскольку элементы матрицы $\hat{A}$ могут быть в равной степени как положительными, так и отрицательными. Таким образом, корреляционная матрица $\hat{C}$ полностью определяет статистические свойства динамической матрицы $\hat{M}$, и при этом плотность колебательных состояний $g(\omega)$ определяется плотностью распределения собственных значений $\rho(\lambda)$ корреляционной матрицы $\hat{C}$. 
Для рассматриваемого случая квадратной решетки распределение $\rho(\lambda)$ имеет вид

$$
\begin{aligned}
\rho(\lambda) & =\frac{1}{4 \pi^{2}} \int_{-\pi}^{\pi} \int_{-\pi}^{\pi} \delta\left(\lambda-4 \Omega^{2}\left(\sin ^{2} \frac{k_{x}}{2}+\sin ^{2} \frac{k_{y}}{2}\right)\right) d k_{x} d k_{y} \\
& =\frac{1}{2 \pi^{2} \Omega^{2}} K\left(\frac{\lambda\left(8 \Omega^{2}-\lambda\right)}{16 \Omega^{4}}\right)
\end{aligned}
$$

где $K(m)=\int_{0}^{\pi / 2}\left[1-m \sin (t)^{2}\right]^{-1 / 2} d t-$ полный эллиптический интеграл первого рода. Для нахождения плотности колебательных состояний $g(\omega)$ рассмотрим производящую функцию моментов от $\rho(\lambda)$ следующего вида

$$
\mathscr{M}(Z)=\sum_{k=1}^{\infty} \frac{1}{Z^{k}} \int \lambda^{k} \rho(\lambda) d \lambda
$$

Для рассматриваемой квадратной решетки можно найти аналитический вид производящей функции моментов

$$
\begin{aligned}
\mathscr{M}(Z) & =\int \frac{\lambda}{Z-\lambda} \rho(\lambda) d \lambda \\
& =\frac{1}{4 \pi^{2}} \int_{-\pi}^{\pi} \int_{-\pi}^{\pi} \frac{4 \Omega^{2}\left(\sin ^{2} \frac{k_{x}}{2}+\sin ^{2} \frac{k_{y}}{2}\right)}{Z-4 \Omega^{2}\left(\sin ^{2} \frac{k_{x}}{2}+\sin ^{2} \frac{k_{y}}{2}\right)} d k_{x} d k_{y} \\
& =\frac{2}{\pi} \frac{Z}{Z-4 \Omega^{2}} K\left(\frac{16 \Omega^{4}}{\left(Z-4 \Omega^{2}\right)^{2}}\right)-1
\end{aligned}
$$

Для любого коррелированного ансамбля Вишарта производящая функция моментов $\mathscr{M}(Z)$ позволяет найти плотность колебательных состояний в виде

$$
g(\omega)=-\frac{2 \omega}{\pi} \operatorname{Im} \frac{1}{Z\left(\omega^{2}\right)},
$$

где конформное отображение $Z(z)$ определяется решением уравнения

$$
Z(\mathscr{M}(Z)+1+\varkappa)=z
$$

относительно $Z$ для заданного $z[32,33]$.

Для каждого значения частоты $\omega$ уравнение (9) может быть решено численным образом, в результате чего получается плотность колебательных состояний, приведенная сплошной линией на рис. 1. Видно, что для каждого значения параметра $\varkappa$ существует некоторая характерная частота $\omega_{c}$, которая разделяет низкочастотное и высокочастотное поведение плотности колебательных состояний.

\section{3. Низкочастотная асимптотика}

Для более подробного изучения плотности колебательных состояний в области частот $\omega \ll \Omega$ можно воспользоваться асимптотикой $\mathscr{M}(Z)$ вблизи $Z=0$

$$
\mathscr{M}(Z)=-1+\frac{Z}{4 \pi \Omega^{2}} \ln \left(-\frac{Z}{32 \Omega^{2}}\right)+\mathscr{O}\left(Z^{2}\right) .
$$

Заметим, что данный вид асимптотики можно обобщить на случай произвольной $\rho(\lambda)$, имеющей постоянное значение при малых $\lambda$, что характерно для любой двумерной системы с линейным законом дисперсии низкочастотных колебаний. В этом случае асимптотику производящей функции моментов можно записать в виде

$$
\mathscr{M}(Z)=-1+\frac{Z}{a} \ln \left(-\frac{Z}{b}\right)+\mathscr{O}\left(Z^{2}\right),
$$

где $a$ и $b-$ константы, имеющие размерность квадрата частоты. Данные константы и связаны с $\rho(\lambda)$ асимптотическим соотношением

$$
\int_{0}^{\infty} \frac{\rho(\lambda)}{x+\lambda} d \lambda=-\frac{1}{a} \ln \frac{x}{b}+\mathscr{O}(x)
$$

при малых $x$. Заметим, что выражение для $a$ и $b$ можно записать в явном виде

$$
a=\frac{1}{\rho(0)}, \quad b=\lambda_{\max } \exp \left(\int_{0}^{\lambda_{\max }} \frac{a \rho(\lambda)-1}{\lambda} d \lambda\right) .
$$

Для описанного выше случая квадратной решетки $a=4 \pi \Omega^{2}, b=32 \Omega^{2}$.

Уравнение (9) с производящей функцией моментов (11) приобретает вид

$$
\varkappa Z+\frac{Z^{2}}{a} \ln \left(-\frac{Z}{b}\right)=\omega^{2} .
$$

Для каждого значения частоты $\omega$ уравнение (14) имеет одно или несколько комплексных решений относительно переменной $Z$. Набор таких точек $Z=X+i Y$ для разных частот $\omega$ образует так называемый критический горизонт в комплексной плоскости [33]. Физически правильным является часть критического горизонта в верхней полуплоскости $(Y>0)$, что соответствует положительной плотности колебательных состояний (8).

\section{4. Изостатический случай $x=0$}

Случаю $\varkappa=0$ соответствует равенство числа степеней свободы $N$ и числа связей $K$. Такой случай в литературе получил название изостатический [6]. В данном случае уравнение (14) можно решить аналитически, что соответствует трем решениям

$$
\begin{aligned}
& Z_{ \pm 1}(\omega)=\sqrt{\frac{2 a \omega^{2}}{W_{ \pm 1}\left(2 a \omega^{2} / b^{2}\right)}} \\
& Z_{0}(\omega)=-\sqrt{\frac{2 a \omega^{2}}{W_{0}\left(2 a \omega^{2} / b^{2}\right)}}
\end{aligned}
$$

где $W_{n}(z)-n$-я ветвь функции Ламберта. Физическим является решение $Z=Z_{-1}$, что обусловлено требованием $\operatorname{Im} Z>0$. Согласно формуле (8), соответствующая 
плотность колебательных состояний имеет вид

$$
g(\omega)=-\frac{1}{\pi} \operatorname{Im} \sqrt{\frac{2}{a} W_{-1}\left(\frac{2 a \omega^{2}}{b^{2}}\right)} .
$$

Заметим, что функция Ламберта $W_{-1}(x)$ при малых $x$ имеет логарифмическую расходимость, которую при $x>0$ можно описать с помощью следующего асимптотического разложения [34]:

$$
W_{-1}(x)=\ln x-\ln (-\ln x)-i \pi+o(1) .
$$

В приведенной выше асимптотике первые два слагаемых расходятся. Остаток асимптотического разложения стремится к 0 при $x \rightarrow 0$ и обозначен как $o(1)$. Основной вклад в расходимость при $x=0$ дает слагаемое $\ln x$, которого достаточно для качественного анализа результатов. Однако для получения более аккуратного результата в последующих выкладках мы будем сохранять оба расходящихся члена. Действительно, при сохранении только первого расходящегося слагаемого относительная погрешность не превосходит $1 \%$ только для чрезвычайно малых значений $x \lesssim 10^{-282}$. Если учесть оба расходящихся слагаемых, то относительная погрешность не превосходит $1 \%$ при $x \lesssim 10^{-6}$. Всюду далее мы будем пренебрегать членами вида $o(1)$ по сравнению с $\ln x$ и $\ln (-\ln x)$. В рамках данного приближения мы можем написать найденное решение $Z(\omega)$ в виде

$$
Z(\omega)=i \sqrt{\frac{2 a \omega^{2}}{L\left(\frac{b^{2}}{2 a \omega^{2}}\right)+i \pi}},
$$

где мы для удобства ввели обозначение $L(x)=\ln x+\ln \ln x$. Не умаляя точности, мы можем получить плотность колебательных состояний в виде

$$
g(\omega)=\sqrt{\frac{2}{a \pi^{2}} L\left(\frac{b^{2}}{2 a \omega^{2}}\right)} .
$$

Полученная плотность колебательных состояний имеет логарифмическую расходимость при малых частотах. Данный результат является особенностью изостатических двумерных неупорядоченных систем. Для трехмерных систем плотность колебательных состояний, полученная в рамках рассмотрения коррелированного ансамбля Вишарта, не расходится при малых частотах [35].

\section{5. Неизостатический случай $x>0$}

При $\varkappa>0$ число связей $K$ превосходит число степеней свободы $N$, что соответствует неизостатическому случаю. В этом случае получить аналитическое решение уравнения для критического горизонта (14) не представляется возможным. Однако мы можем получить ответ в том же приближении, в котором написаны формулы (18) и (20). Для этого запишем уравнение (14) в следующем виде

$$
Z=\frac{\sqrt{a \omega^{2}}}{\sqrt{\frac{a \varkappa^{2}}{4 \omega^{2}}}+\sqrt{\frac{a \varkappa^{2}}{4 \omega^{2}}+\ln \left(-\frac{Z}{b}\right)}}
$$

Мы можем найти сколь угодно точное приближение, используя метод простых итераций. В качестве нулевого приближения возьмем полученную ранее зависимость $Z(\omega)$ в изостатическом случае $\varkappa=0$ :

$$
Z^{(0)}(\omega)=i \sqrt{\frac{2 a \omega^{2}}{L\left(\frac{b^{2}}{2 a \omega^{2}}\right)+i \pi}} .
$$

Последующие итерации для нахождения $Z(\omega)$ имеют вид

$$
Z^{(n+1)}(\omega)=\frac{\sqrt{a \omega^{2}}}{\sqrt{\frac{a \varkappa^{2}}{4 \omega^{2}}}+\sqrt{\frac{a \varkappa^{2}}{4 \omega^{2}}+\ln \left(-\frac{Z^{(n)}(\omega)}{b}\right)}}
$$

После двукратного применения итерационной процедуры и пренебрежения членами вида $o(1)$ по сравнению c $\ln x$ и $\ln \ln x$, мы получаем следующее выражение для критического горизонта $Z(\omega)$ :

$$
Z(\omega)=\frac{\sqrt{2 a \omega^{2}}}{\sqrt{\frac{a \varkappa^{2}}{2 \omega^{2}}}+\sqrt{\frac{a \varkappa^{2}}{2 \omega^{2}}-L\left(\frac{b^{2}}{2 a \omega^{2}}\right)-2 \pi i}},
$$

Последующее применение итерационной процедуры (23) не меняет полученного выражения в рамках использованного приближения.

В результате, мы получаем следующее выражение для плотности колебательных состояний

$$
g(\omega)=\frac{\sqrt{2}}{\pi \sqrt{a}} \operatorname{Im} \sqrt{f(\omega)-2 \pi i},
$$

где для удобства введено обозначение

$$
f(\omega)=\frac{a \varkappa^{2}}{2 \omega^{2}}-L\left(\frac{b^{2}}{2 a \omega^{2}}\right) .
$$

Полученный ответ можно записать без использования комплексных чисел

$$
g(\omega)=\frac{1}{\pi \sqrt{a}} \sqrt{\sqrt{f(\omega)^{2}+4 \pi^{2}}-f(\omega)} .
$$

При $\varkappa=0$ формула (27) переходит в полученную ранее формулу (20). Рис. 1 показывает хорошее совпадение полученной формулы с точным значением плотности колебательных состояний (8), полученной путем численного решения комплексного уравнения (9). 


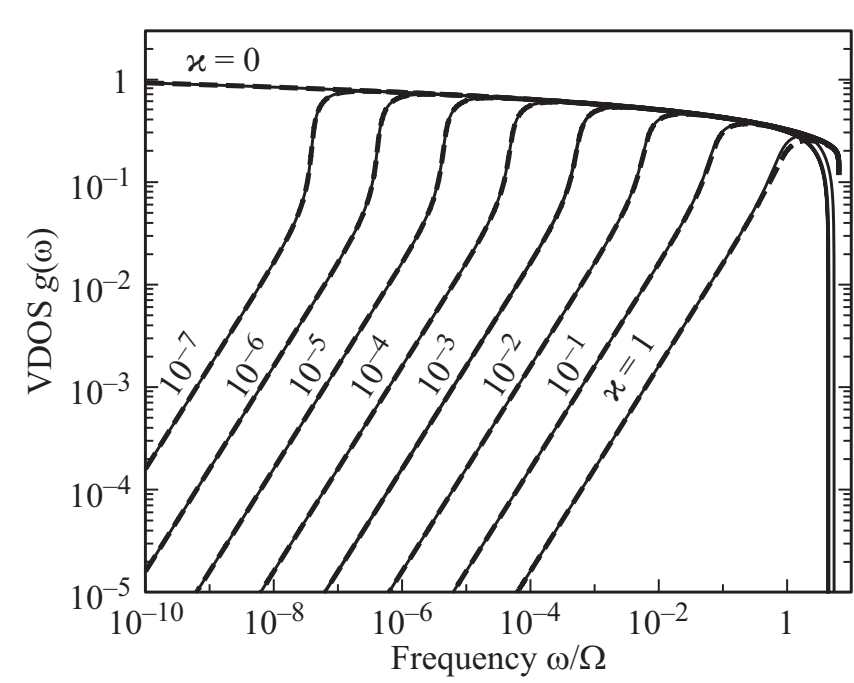

Рис. 1. Плотность колебательных состояний в коррелированном ансамбле Вишарта. Тонкими сплошными линиями показано точное решение, построенное по формуле (8) для соответствующих значений параметра $\varkappa$. Штриховыми линиями показано асимптотическое выражение (27) с параметрами $a=4 \pi \Omega^{2}, b=32 \Omega^{2}$.

\section{6. Кроссовер}

Если $\varkappa>0$, то имеется две области плотности колебательных состояний, разделенных узким и быстро возрастающим участком $g(\omega)$ (рис. 1). Справа от переходной области плотность колебательных состояний близка к изостатической $(\varkappa=0)$. Слева от переходной области наблюдается режим $g(\omega) \sim \omega$, выглядящий как набор параллельных линий на рис. 1. Переходному участку (кроссоверу) соответствует смена знака функции $f(\omega)$. Частота кроссовера $\omega_{c}$ определяется уравнением $f(\omega)=0$ и в рамках используемых приближений может быть записана в виде

$$
\omega_{c}=\frac{\varkappa}{2} \sqrt{\frac{a}{L(2 b / a \varkappa)}} .
$$

В области частот $\omega<\omega_{c}$ функция $f(\omega)$ положительна. При этом в данной области выполняется соотношение $f(\omega) \gg 2 \pi$, кроме узкой области $\left|\omega-\omega_{c}\right| \lesssim \omega_{c} / L(2 b / a \varkappa)$. В этом случае мы можем воспользоваться разложением $\sqrt{f(\omega)^{2}+4 \pi^{2}} \simeq$ $\simeq f(\omega)+2 \pi^{2} / f(\omega)$ и получить плотность состояний в виде

$$
g_{l}(\omega)=\sqrt{\frac{2}{a f(\omega)}} .
$$

Поскольку $f\left(\omega_{c}\right)=0$, то $\omega_{c}$ является частотой, при которой асимптотика $g_{l}(\omega)$ неограниченно возрастает.

В области низких частот $\omega \ll \omega_{c}$ функция $f$ принимает вид $f(\omega) \simeq a \varkappa^{2} / 2 \omega^{2}$, в результате чего

$$
g(\omega)=\frac{2 \omega}{a \varkappa}+O\left(\omega^{2}\right)
$$

Этот результат согласуется с законом Дебая для фононов $g_{D}(\omega)=\omega /(2 \pi E)$, где $E-$ модуль Юнга системы, в которой мы для простоты полагаем, что массы частиц единичны и на единицу площади приходится одна степень свободы. Можно предполагать, что вся область левее частоты кроссовера $\omega<\omega_{c} \ll \Omega$ описывает область слабо рассеивающихся длинноволновых колебаний с примерно линейным законом дисперсии.

Из сравнения формулы (30) с законом Дебая можно найти модуль Юнга

$$
E=\frac{a \varkappa}{4 \pi} .
$$

Для параметров $a=4 \pi \Omega^{2}$ и $b=32 \Omega^{2}$ модуль Юнга приобретает вид $E=\Omega^{2} \varkappa$. Результат (31) хорошо согласуется с правилом Максвелла, согласно которому жесткость системы $E=0$ при равенстве числа степеней свободы и числа связей системы $N=K$. Заметим, что выражение (31) получено точно для любого значения $\varkappa$, несмотря на сделанные ранее приближения.

В области частот $\omega_{c}<\omega \ll \Omega$ функция $f(\omega)$ отрицательна. При этом в данной области выполняется соотношение $|f(\omega)| \gg 2 \pi$ кроме узкой области $\left|\omega-\omega_{c}\right| \lesssim \omega_{c} / L(2 b / a \varkappa)$. В этом случае мы можем пренебречь слагаемым $4 \pi^{2}$ в формуле (27) и получить плотность состояний в виде

$$
g_{r}(\omega)=\frac{1}{\pi} \sqrt{-\frac{2}{a} f(\omega)} .
$$

Заметим, что $\omega_{c}$ является частотой, при которой асимптотика $g_{r}(\omega)$ обращается в ноль.

На рис. 2 показано сравнение асимптотических выражений приведенной плотности состояний $g_{l}(\omega) / g_{D}(\omega)$

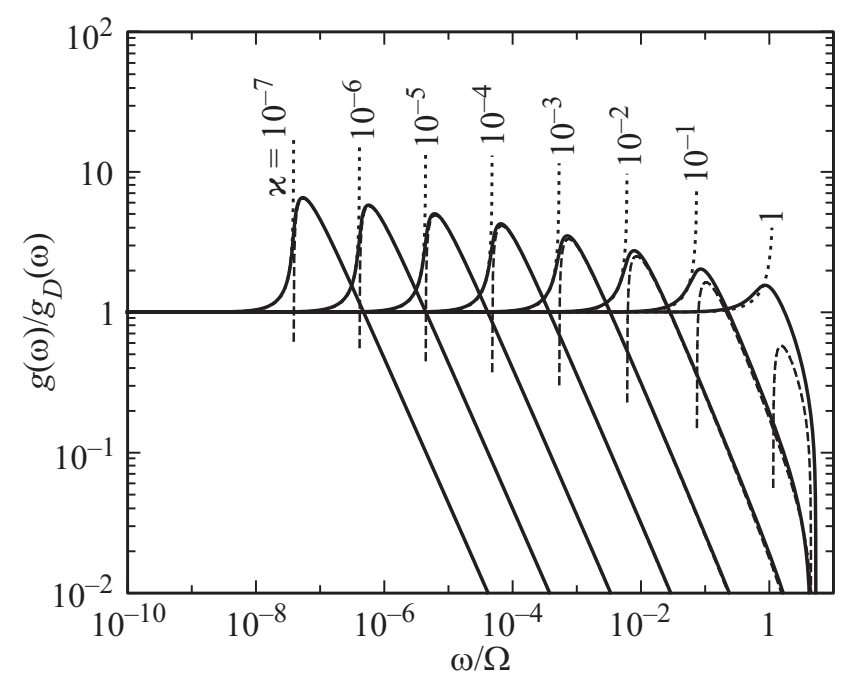

Рис. 2. Бозонный пик в коррелированном ансамбле Вишарта. Тонкими сплошными линиями показано точное решение, построенное по формуле (8) для соответствующих значений параметра $\varkappa$. Пунктирными линиями показано асимптотическое выражение (29), а штриховыми - выражение (32) с параметрами $a=4 \pi \Omega^{2}, b=32 \Omega^{2}$. 
и $g_{r}(\omega) / g_{D}(\omega)$ с точным значением $g(\omega) / g_{D}(\omega)$, полученным по формуле (8) путем численного решения комплексного уравнения (9). Видно, что обе асимптотики хорошо согласуются с точным решением кроме узкой области вблизи частоты кроссовера $\omega=\omega_{c}$.

\section{7. Бозонный пик}

На рис. 2 наблюдается максимум приведенной плотности состояний $g(\omega) / g_{D}(\omega)$, который в литературе известен как бозонный пик. Частота бозонного пика $\omega_{b}$ незначительно превосходит частоту кроссовера $\omega_{c}$ и может быть найдена как частота, при которой соответствующая производная обращается в ноль

$$
\left.\frac{d}{d \omega} \frac{g(\omega)}{g_{D}(\omega)}\right|_{\omega=\omega_{b}}=0 .
$$

Данное уравнение может быть сведено к уравнению вида

$$
2 \sqrt{f^{2}+4 \pi^{2}}+\left.\omega f^{\prime}(\omega)\right|_{\omega=\omega_{b}}=0 .
$$

В рамках используемых приближений решением уравнения (34) является

$$
\omega_{b}=\varkappa \sqrt{\frac{a}{1+2 L(\sqrt{2} b / a x)}} .
$$

Заметим, что $\omega_{b} \approx \sqrt{2} \omega_{c}$ в рамках более грубого приближения, в котором можно считать, что $L(x) \approx \ln x$. Таким образом, частота бозонного пика по порядку величины совпадает с частотой кроссовера $\omega_{c}$.

В результате мы можем установить связь между частотой бозонного пика $\omega_{b}$ и жесткостью системы $E$

$$
\omega_{b}=\frac{4 \pi E}{\sqrt{a(1+2 L(\sqrt{2} b / 4 \pi E))}} .
$$

Таким образом, связь между частотой бозонного пика $\omega_{b}$ и жесткостью системы $E$ имеет логарифмическую поправку к линейному закону, что является особенностью двумерных систем. В трехмерных системах наблюдается линейный закон без логарифмических поправок [35].

Определим высоту бозонного пика как максимум приведенной плотности состояний $h=\max \left[g(\omega) / g_{D}(\omega)\right]$ $=g\left(\omega_{b}\right) / g_{D}\left(\omega_{b}\right)$. Используя формулу $(27)$ и полученное значение частоты бозонного пика $\omega_{b}(36)$, в рамках использованных приближений мы получаем высоту бозонного пика

$$
h=\frac{1}{\pi} L\left(\frac{\sqrt{2} b}{a \varkappa}\right) .
$$

Таким образом, высота бозонного пика увеличивается логарифмически при уменьшении параметра $\varkappa$ (т.е. при увеличении беспорядка), что согласуется с полученными ранее численными результатами [36,37]. Данная зависимость отличается от трехмерного случая, в котором высота бозонного пика растет степенным образом при увеличении беспорядка [26].

\section{8. Заключение}

В работе показано, что коррелированный ансамбль Вишарта естественным образом описывает колебательные свойства аморфных твердых тел и учитывает их механическую устойчивость и правило сумм. Данный ансамбль позволяет установить аналитические выражения, описывающие асимптотическое поведение плотности колебательных состояний на низких частотах для произвольной двумерной системы с линейным законом дисперсии низкочастотных колебаний. Данные формулы подтверждаются точным решением для случая квадратной решетки, для которой было получено аналитическое выражение производящей функции моментов $\mathscr{M}(Z)$.

Основным параметром модели является безразмерный параметр $\varkappa$, который позволяет менять степень беспорядка системы в неограниченных пределах. В случае малых значений $\varkappa \ll 1$ система обладает существенным беспорядком и демонстрирует избыточную плотность колебательных состояний $g(\omega)$ по сравнению с дебаевской плотностью состояний $g_{D}(\omega)$. В данном случае наблюдается бозонный пик в приведенной плотности колебательных состояний $g(\omega) / g_{D}(\omega)$, высота которого, в отличие от трехмерных систем, растет логарифмически при уменьшении параметра $\varkappa$.

Показано, что в изостатическом случае $\varkappa=0$ частота бозонного пика $\omega_{c}$ и жесткость системы $E$ стремятся к нулю. При этом плотность колебательных состояний двумерных систем обладает логарифмической расходимостью при малых частотах. Связь между частотой бозонного пика $\omega_{b}$ и жесткостью системы $E$ имеет логарифмическую поправку к линейному закону.

\section{Благодарности}

Авторы благодарят Совет по грантам Президента Российской Федерации за финансовую поддержку (проект МК-3052.2019.2).

\section{Конфликт интересов}

Авторы заявляют, что у них нет конфликта интересов.

\section{Список литературы}

[1] V.L. Gurevich, D.A. Parshin, H.R. Schober. Phys. Rev. B 67, 094203 (2003).

[2] V.K. Malinovsky, A.P. Sokolov. Solid State Commun. 57, 757 (1986).

[3] P. Benassi, M. Krisch, C. Masciovecchio, V. Mazzacurati, G. Monaco, G. Ruocco, F. Sette, R. Verbeni. Phys. Rev. Lett. 77, 3835 (1996).

[4] A. Wischnewski, U. Buchenau, A.J. Dianoux, W.A. Kamitakahara, J.L. Zarestky. Phil. Mag. B 77, 579 (1998).

[5] S. Kojima, Y. Matsuda, M. Kodama, H. Kawaji, T. Atake. Chinese J. Phys. 49, 414 (2011).

[6] C.S. O’Hern, L.E. Silbert, A.J. Liu, S.R. Nagel. Phys. Rev. E 68, 011306 (2003). 
[7] A.J. Liu, S.R. Nagel. Annu. Rev. Condens.Matter Phys. 1, 347 (2010).

[8] Y. Nie, H. Tong, J. Liu, M. Zu, N. Xu. Front. Phys. 12, 126301 (2017).

[9] L. Zhang, J. Zheng, Y. Wang, L. Zhang, Z. Jin, L. Hong. Y. Wang, J. Zhang. Nature Commun. 8, 67 (2017).

[10] Y. Wang, L. Hong, Y. Wang, W. Schirmacher, J. Zhang. Phys. Rev. B 98, 174207 (2018).

[11] W. Steurer, A. Apfolter, M. Koch, W.E. Ernst, B. Holst, E. Sondergard, J.R. Manson. Phys. Rev. Lett. 99, 3, 035503 (2007).

[12] S. Kojima, M. Kodama. Phys. B 263-264, 336 (1999).

[13] V. Vitelli, N. Xu, M. Wyart, A.J. Liu, S.R. Nagel. Phys. Rev. E 81, 021301 (2010).

[14] R. Speicher, C. Vargas. Random Matrices: Theory and Applications 1, 1150008 (2012).

[15] J. Harnad. Random Matrices, Random Processes and Integrable Systems Springer N. Y. (2011).

[16] M. Prahofer, H. Spohn. Phys. Rev. Lett. 84, 4882 (2000).

[17] A.M. Tulino, S. Verdu. Found. Trends Commun. Inf. Theory 1, 1 (2004).

[18] K.E. Wage. J. Acoust. Soc. Am. 138, 1840 (2015).

[19] A. Edelman, B. Sutton, Y. Wang. Proc. Symp. in Appl. Math. 72, 53 (2014).

[20] L. Laloux, P. Cizeau, M. Potters, J-P. Bouchaud. Int. J. Theor. Appl. Finance 3, 391 (2000).

[21] H. Meyer, J.C. Angles d'Auriac. Phys. Rev. E 55, 6608 (1997).

[22] T. Guhr, A. Mueller-Groeling, H.A. Weidenmueller. Phys. Rep. 299, 189 (1998).

[23] S. Jalan, J.N. Bandyopadhyay. Phys. Rev. E 76, 046107 (2007).

[24] K. Rajan, L.F. Abbott. Phys. Rev. Lett. 97, 188104 (2006).

[25] Я.М. Бельтюков, Д.А. Паршин. ФТТ 53, 142 (2011).

[26] Я.М. Бельтюков, Д.А. Паршин. Письма в ЖЭТФ 104, 570 (2016).

[27] T.S. Grigera, V. Martin-Mayor, G. Parisi, P. Verrocchio. J. Phys.: Condens. Matter 14, 2167 (2002).

[28] M.L. Manning, A.J. Liu. Europhys. Lett. 109, 36002 (2015).

[29] Y.M. Beltukov. JETP Letters 101, 345 (2015).

[30] M. Baggioli, R. Milkus, A. Zaccone. Phys. Rev. E 100, 062131 (2019).

[31] Y.M. Beltukov, V.I. Kozub, D.A. Parshin. Phys. Rev. B 87, 134203 (2013).

[32] Z. Burda, A. Gorlich, A. Jarosz, J. Jurkiewicz. Physica A 343, 295 (2004).

[33] Z. Burda, A. Gorlich, J. Jurkiewicz, B. Waclaw. Eur. Phys. J. B 49, 319 (2006).

[34] R.M. Corless, G.H. Gonnet, D.E. Hare, D.J. Jeffrey, D.E. Knuth. Adv. Comp. Math. 5, 329-359 (1996).

[35] D.A. Conyuh, Y.M. Beltukov. J. Phys. Conf. Ser. 1391, 012118 (2019).

[36] D.A. Conyuh, Y.M. Beltukov, D.A. Parshin. J. Phys. Conf. Ser. 929012031 (2017).

[37] Д.А. Конюх, Я.М. Бельтюков, Д.А. Паршин. ФТТ 60, 369 (2018).

Редактор Т.Н. Василевская 